\section{Cahiers de Narratologie}

Analyse et théorie narratives

$10.2 \mid 2001$

La voix narrative

\title{
Questions de prosimétrie dans le Satiricon de Pétrone : la figure d'Encolpe come narrateur poète
}

\section{Géraldine Puccini}

\section{(2) OpenEdition}

1 Journals

Édition électronique

URL : http://journals.openedition.org/narratologie/10269

DOI : 10.4000/narratologie.10269

ISSN : 1765-307X

Éditeur

LIRCES

\section{Édition imprimée}

Date de publication : 1 janvier 2001

Pagination : 383-393

ISBN : 2914561032

ISSN : $0993-8516$

\section{Référence électronique}

Géraldine Puccini, «Questions de prosimétrie dans le Satiricon de Pétrone : la figure d’Encolpe come narrateur poète », Cahiers de Narratologie [En ligne], 10.2 | 2001, mis en ligne le 01 janvier 2001, consulté le 11 juin 2020. URL : http://journals.openedition.org/narratologie/10269 ; DOI : https:// doi.org/10.4000/narratologie.10269 


\title{
QUESTIONS DE PROSIMÉTRIE DANS LE SATIRICON DE PÉTRONE : LA FIGURE D'ENCOLPE COMME NARRATEUR POÈTE.
}

\author{
Géraldine PUCCINI \\ Université de Nice-Sophia Antipolis
}

Sur trente-quatre poèmes conservés dans le Satiricon de Pétrone, dont quatre citations de Virgile et une de l'auteur de mimes Publilius Syrus, treize poèmes peuvent être attribués au narrateur principal Encolpe. Ils se situent après l'épisode de la Cena Trimalchionis et se regroupent en trois sections distinctes : deux poèmes s'insèrent dans le récit de la rivalité amoureuse entre Encolpe et son ami Ascylte à propos de leur esclave Giton ${ }^{1}$; huit poèmes émaillent l'histoire d'amour entre Encolpe et la belle Circé ${ }^{2}$; les trois derniers poèmes concernent l'épisode où Encolpe se trouve aux prises avec une prêtresse de Priape, Oenothée.

Encolpe, narrateur homodiégétique, choisit donc d'insérer dans la trame de sa prose narrative des poèmes, bien qu'il ne se présente absolument pas comme un poeta, ce rôle professionnel étant dévolu à un autre personnage, Eumolpe. Certes, la fiction romanesque antique n'est pas un genre défini, régi par un code et des règles rigides, ce qui lui permet de pouvoir recourir en toute liberté à la prosimétrie, typique de la satire ménippée à laquelle Pétrone semble beaucoup emprunter, mais l'espace de la narration est particulièrement occupé par la poésie dans le Satiricon et l'auteur Pétrone oscille sans cesse entre être un romancier ou un poète. Rappelons que sur vingt-huit œuvres parvenues jusqu'à nous que nous classons sous le terme anachronique de "roman» (sept romans complets ou presque et vingt et un fragments),

1 Sat., 79, $7 ; 80,9 ; 82,5$.

2 Sat., 126, $18 ; 127,9 ; 128,6 ; 131,8 ; 132,8 ; 132,11 ; 132,15$; $133,2$. 
trois seulement recourent à la prosimétrie : en latin, l'œuvre de Pétrone, en langue grecque, le fragment de Iolaos et le fragment de Tinouphis ${ }^{3}$. Nous analyserons donc la tension propre au Satiricon que crée l'alternance de la prose avec la poésie, structures rivales de la représentation, et les raisons pour lesquelles le narrateur Encolpe se fait à certains moments privilégiés poète.

Si l'irruption du poème dans la narration vient perturber le rythme de celle-ci, l'élément poétique introduit par le narrateur est toutefois en relation étroite avec le cadre narratif : tel un miroir, il vient redoubler l'histoire racontée en prose. Mais le miroir est toujours déformant, comme si la poésie était un moyen de dénoncer les illusions du narrateur, à peine esquissées par le récit en prose, en jouant sur les contrastes et les ruptures. C'est ainsi que le premier poème déclamé par Encolpe, constitué de cinq hendécasyllabes ${ }^{4}$, constitue une insertion lyrique, exagérément hyperbolique, destinée à célébrer la volupté extrême que le narrateur connut dans les bras de Giton, avant que celui-ci ne le trahisse et ne choisisse Ascylte pour amant, abandonnant Encolpe à sa douleur et à son humiliation. Si le choix de la forme poétique permet au narrateur de magnifier sa relation avec Giton, d'isoler des autres cette nuit extraordinaire par l'ardeur de leurs étreintes, il lui permet aussi d'intensifier la cruelle déconvenue qui s'ensuivit. L'alternance de la prose et de la poésie est un

${ }^{3}$ Le Satiricon est peut-être à mettre en relation avec un genre établi de fiction grecque prosimétrique non idéalisante, auquel appartiennent peut-être le fragment de Iolaus et le fragment de Tinouphis qui utilisent à la fois prose et poésie. Voir S. A. Stephens and J.-J. Winkler, Ancient Greek Novels, The Fragments, Princeton University Press, Princeton, New Jersey, 1995, p. 364.

4 Dans le carmen 42 de Catulle, le poète compare ses hendécasyllabes à des chiens de chasse ou de garde en fureur qu'il lâche contre une prostituée, parce qu'elle refuse de lui rendre ses lettres d'amour elles-mêmes écrites selon le même mètre. La plus grande partie du poème est virulente et injurieuse, mais les derniers vers effectuent une palinodie ironique, encore plus insultante pour une femme décriée : la moecha turpis, la moecha putida devient pudica et proba. L'hendécasyllabe est à la fois utilisé pour le blâme et l'éloge ironique. 
moyen efficace de marquer les écarts au point même que les événements sont ressentis de manière totalement théâtrale, avec une exagération qui les vide de leur signification pathétique. Le mouere est mis à mal au profit du delectare, et même au profit de la dérision. Le narrateur lui-même, conscient du grotesque de la situation, tourne son ironie contre lui et sa crédulité par trop naïve : aussitôt après son poème, il affirme malicieusement que c'est "à tort qu'<il se> réjouit » ${ }^{5}$. Le narrateur ôte de lui-même tout crédit à sa poésie : le lyrisme tombe à plat dans le prosaïsme cocasse de la narration en prose qui suit, où le narrateur occupe une position particulièrement dégradée.

D'ailleurs, le vers était dans le fruit, car le poème construit ironiquement lui-même la cassure de l'idéalisation qu'il projette ostensiblement. C'est ainsi que les errantes animas, les " souffles défaillants » des deux amants annoncent ironiquement l'errance de Giton d'un amant à un autre et que le dernier vers du poème est à double entente : Ego sic perire coepi, "c'est ainsi que je me sentis mourir », mourir de plaisir, mais aussi bientôt mourir de honte et de chagrin avec la tentation du suicide, une fois que Giton aura choisi Ascylte pour amant. La poésie devient ici l'instrument privilégié de la mise à mal du narrateur tourné en ridicule ; l'univers imaginaire de l'œuvre est d'autant plus dégradé que le poème, de manière tout ironique, en souligne le grotesque et le dérisoire.

Le second poème composé de distiques élégiaques clôt cet épisode par une dénonciation de l'amitié, incapable d'être désintéressée et sincère. Ce lieu commun rhétorique peut être attribué au narrateur Encolpe. Mais, comme aucune formule d'introduction ne le précède, comme le « je » n'y apparaît pas et qu'il s'adresse de manière générale à ceux qui se prétendent amis, il pourrait tout aussi bien être dit par une sorte de " voix off », impersonnelle, venant commenter la mésaventure d'Encolpe de manière sentencieuse et théâtrale. D'ailleurs, la rivalité triangulaire dont Encolpe est la victime est une véritable scène de comédie, un mime où les trois protagonistes jouent des « rôles comiques »:

5 Sat., 79, 8 : sine causa gratulor mihi. 
Grex agit in scaena mimum: pater ille uocatur, filius hic, nomen diuitis ille tenet. Mox ubi ridendas inclusit pagina partes, uera redit facies, adsimulata perit.

« La troupe de théâtre joue sur scène un mime : celui-là fait le père, celui-ci le fils, cet autre tient le rôle du riche.

Puis, quand la page se referme sur leurs rôles comiques, leur vrai visage réapparaît, le masque tombe. »

Méta-commentaire, ce poème en forme d'épigramme indique comment interpréter l'épisode : la fiction est à mettre en relation avec le mime farcesque ${ }^{6}$ et le narrateur y joue le rôle de victime pitoyable et comique. La vie est une scène de théâtre, les personnages sont des acteurs ; et ce qu'ils jouent est un mime, pas mểme une comédie. Le poème dévoile la conscience que possède le Satiricon de sa propre fictionnalité et dénonce en même temps aux yeux du lecteur ses duperies et ses travestissements. Il ne fait que renforcer le thème de la trahison déjà exploité par le récit en prose et permet de passer d'un cas particulier à une situation générale. Ce second poème est lié au premier, dans la mesure où tous deux semblent signaler les frontières d'un épisode, dont ils marquent le début et la fin. Le second vient dénoncer l'illusion développée par le premier et rend dérisoire la passion d'Encolpe pour Giton. Lui-même, en tant que déclamation emphatique d'Encolpe sur son destin et ses malheurs, tourne au ridicule : Encolpe vit son désespoir sur le mode de la grandiloquence et du spectacle ; il habille le dramatique en théâtral. Du coup, même sa souffrance en devient inauthentique et factice.

Cette structure où les poèmes se répondent est retrouvée dans l'épisode suivant. C'est à Crotone, lors de ses amours contrariées avec une belle dame du nom de Circé, que onze poèmes vont être prononcés par Encolpe. Le premier est un «madrigal» de trois distiques élégiaques composé en l'honneur de la beauté de Circé qui ne fait que redoubler et

6 En grec, on ne trouve la prosimétrie intégrée dans le texte que dans le mime. Cf. S. A. Stephens and J.-J. Winkler, Ancient Greek Novels, The Fragments, p. 364. 
amplifier l'éloge de cette beauté surhumaine qu'Encolpe vient de faire dans son récit en prose. La redondance ainsi produite est au service d'un style asianiste dont la copia uerborum est une caractéristique essentielle.

Le poème s'insère parfaitement dans le cadre narratif dont il amplifie le contenu de manière si dithyrambique que cela finit là aussi par sonner faux et ridicule. Sous prétexte de magnifier ce que le récit en prose semble impuissant à réaliser, il réduit à néant l'effet que le récit en prose aurait pu produire seul sur le lecteur. La beauté de Circé, comme celle de toutes les héroïnes de fiction antique, est incommensurable : " Aucun mot ne pourrait décrire sa beauté et, quoique je dise, je serai en-deçà de la réalité », avoue le narrateur qui n'en donne pas moins une description succincte, concluant que « tout cela aurait éclipsé l'éclat du marbre de Paros » ${ }^{7}$. Le poème apostrophe alors dans une indignation comique le roi des dieux, Jupiter, devenu fabula muta, « un personnage muet » devant la beauté de Circé : "voici la véritable Danaé. Essaie seulement de toucher ce corps et bientôt une ardente chaleur fera fondre tes membres. " Le substantif fabula introduit de nouveau une atmosphère théâtrale : le narrateur, se faisant poeta, invite à une lecture théâtrale de sa fiction romanesque et montre qu'il vit même l'amour sur le mode théâtral. Quant à l'image grotesque du dieu se liquéfiant de désir devant Circé, ce qui détruit totalement le sublime possible de l'écriture prosaïque, elle annonce ironiquement l'amollissement prochain d'Encolpe, incapable de satisfaire sexuellement sa belle...

L'éloge de la beauté de Circé se poursuit par la description de la pelouse en 127, 9, où la jeune femme invite Encolpe à s'allonger pour des ébats à l'abri des regards indiscrets. « La terre revêtue de fleurs variées » devient le sujet d'un petit poème de sept hexamètres dactyliques parodiant un passage de l'Iliade ${ }^{8}$. L'éclat des roses, des violettes, du souchet et des lys compose un paysage idyllique où la nature se met au service de l'amour, la lumière du jour se faisant même plus brillante pour favoriser cet «amour secret». Mais une fois

7 Sat., 125, 14-18.

8 Homère, Iliade, XIV, 346 et suiv. 
encore, le poème est là pour mettre en valeur le décalage qui va suivre entre l'idéal et la réalité sordide et grotesque. S'il parodie une scène épique mettant en scène le hieros gamos de Zeus et d'Héra sur le mont Ida, c'est pour mieux grossir l'échec sexuel humiliant qu'Encolpe subit auprès de Circé. Neuf hexamètres dactyliques en 128, 6 viennent apporter une conclusion à cet épisode, développant l'idée émise par Encolpe dans sa narration : n'aurait-il pas été le jouet d'une vision? Les échos entre les deux poèmes sont multiples. Le dies du premier poème devient dans le second une nox soporifera ; la terra parens, la terre mère, devient effossa tellus, une terre creusée ; les jeux amoureux entre Encolpe et Circé sont remplacés par les jeux des songes (le verbe ludere est utilisé dans les deux situations) ; les flammes qui embrasent la poitrine de Jupiter se métamorphosent en " or", en « trésors » qui alourdissent le " giron » du voleur ; le « secret amour » est remplacé par «l'or secret ». Enfin, le huitième hexamètre du poème 128,6 reprend le dernier vers du poème 80, 6 : ueraque forma redit fait écho à uera redit facies, dénonçant les illusions successives auxquelles se laisse prendre le narrateur. Comme le poème 80,9 sur le pouvoir de Fortuna et sur la fausseté de l'amitié qui tenait lieu de conclusion à la perte de Giton, ce poème masque lui aussi par un discours pseudo-moralisateur et sentencieux les véritables raisons de la fuite de Circé.

Le poème 131,8 , composé de huit hexamètres dactyliques, reprend la même thématique que le poème 127,9 : il campe un locus amoenus traditionnel, explicitement désigné comme un dignus amore locus, un « lieu fait pour l'amour ». Il intervient de la même manière dans la narration : il précède les ébats amoureux des deux jeunes gens, mais cette fois-ci, ils sont couronnés de succès. Le texte très lacunaire laisse ensuite entendre que Circé doit subir plusieurs affronts de la part d'Encolpe, souvent impuissant à la satisfaire. Elle finit, pour s'en venger, par le faire battre par ses esclaves et par le faire jeter à la rue. Pour sauver la face, il simule un malaise et, caché au fond de son lit, tourne le feu de sa fureur contre la partie de son corps qui est l'auteur de tous ses malheurs. Il lui adresse neuf vers sotadiques en 132,8 . 
Dans une parodie de style épique, notamment de Virgile9, nous passons des roses, des violettes, du souchet, des lys, du platane, du laurier, du cyprès et des pins, énumérés dans les poèmes précédents, au chou et à sa mollesse ! Encolpe mime de manière burlesque un combat contre son sexe, mais on ne sait qui du sexe qui, " plus glacé que le froid de l'hiver, roide de peur, s'était réfugié dans <ses $>$ entrailles et se dissimulait dans leurs mille replis » ou de la main «tremblante » sachant mal utiliser " la terrible hache à deux tranchants » est le plus peureux et le plus lâche. Incapable de châtier l'objet de sa fureur, Encolpe se contente de quelques paroles vexatoires, mais, empruntant divers passages à Virgile ${ }^{10}$ pour former trois hexamètres dactyliques, il en dénonce toute l'inanité :

« tandis que j'épanchais ces paroles pleines de colère,

Lui, se détournant, tenait les yeux fixés au sol, et son visage ne trahit pas plus de trouble à ce discours que les saules flexibles ou les pavots à la tige lasse. »

Tout au long du roman, le narrateur participe à la parodie du style épique. Le comportement de Pétrone vis-à-vis de Virgile est analogue à celui de l'empereur Néron « rejouant » Auguste de manière parodique, comme l'atteste Suétone'l. Pour reprendre une belle formule de Catherine Connors ${ }^{12}$, Auguste est le poète épique de l'empire et Néron, le romancier qui le parodie, brisant le lien impérial avec le passé épique. Encolpe, par certains de ses poèmes, transporte le monde de l'épopée dans un contexte dégradé pour aboutir à une

${ }^{9}$ Cf. Virgile, Enéide, II, 479.

10 Les deux premiers vers reprennent les vers $469-470$ du livre VI de l'Enéide, le dernier est formé de deux hémistiches, empruntés le premier à l'Eglogue V, vers 16, le second, à l'Enéide IX, vers 436.

11 Suétone, Nero., 25, 1 : Néron fait son entrée à Rome « sur le char qui avait servi autrefois pour le triomphe d'Auguste, vêtu de pourpre, avec une chlamyde parsemée d'étoiles d'or, la couronne olympique sur la tête, et la couronne pythique à la main droite, précédé d'un cortège portant ses autres couronnes, avec des pancartes qui mentionnaient en quel lieu, de quels concurrents, pour quel chant ou pour quelle pièce il avait triomphé » (traduction d'Henri Ailloud, Paris, Les Belles Lettres, 1967).

12 C. Connors, Petronius the poet. Verse and literary tradition in the Satyricon, Cambridge University Press, 1998, p. 5. 
parodie burlesque, telle qu'elle a pu exister sur scène dans certains mimes qui recouraient à des gestes lascifs et à un langage sexuel vulgaire.

Les vers sotadiques utilisés par Encolpe le sont également au chapitre 23, 3 par un cinaedus chargé par Quartilla de redonner vigueur au narrateur. Le recours à ce mètre est un autre moyen de parodier le style épique. Comme le fait remarquer Catherine Connors ${ }^{13}$, cette forme métrique est intimement liée à son contenu : de même que le cinaedus, en tant qu'homosexuel passif, inverse les normes du comportement sexuel des hommes libres, de même, le vers sotadique inverse les structures normatives de l'hexamètre. La masculinité épique est rabaissée à la fois par le sujet du poème - Encolpe essaie par trois fois de saisir son sexe pour le châtier, comme Enée a tenté à trois reprises d'étreindre les ombres des morts aimés aux Enfers - et par le choix du mètre lui-même ${ }^{14}$.

Par les trois hexamètres dactyliques de 132,11 , le narrateur, cette fois-ci, morcelle la matière épique pour la remodeler à sa façon et en fabriquer une fiction inédite ${ }^{15}$. Comme l'a bien montré Catherine Connors ${ }^{16}$, Pétrone cherche consciemment à représenter ce processus de fragmentation, de remodelage et de reconnaissance que constitue la parodie. L'irruption d'un tel montage de citations virgiliennes dans un contexte obscène est un exemple extrême de ce processus à l'œuvre dans le Satiricon. Il a donc avant tout une signification métapoétique : il est la métaphore de cette métamorphose parodique de l'épopée en fiction burlesque obscène.

Seulement, la parodie a un but ambivalent et complexe : soit elle affiche sa connaissance et son respect du modèle qu'elle imite et renforce alors sa position dominante, soit elle est un processus de transgression qui remet en cause cette

13 Ibid., p. 31. Elle émet l'hypothèse qu'il aurait existé des transformations d'hexamètres épiques en vers sotadiques.

${ }^{14}$ Le fragment versifié de Iolaos est en vers sotadiques et le sujet en est lui aussi obscène. Cette forme métrique était couramment associée aux galli, eux-mêmes identifiés à des cinaedi : voir Demetrios, De eloc., 189 ; Syrianus, 1, 47, 9 (Rabe).

15 Trimalchion fait de même en 50, 5-6, l'esclave Massa, en 68, 5.

16 Ibid., pp. 21-24. 
position. Si l'on suit la théorie de Bakhtine ${ }^{17}$, le rire abolit toute distance hiérarchisante, détruit la peur et la piété éprouvées devant un objet qu'il rend familier, au point de pouvoir l'explorer de manière totalement libre. Bien sûr, les poèmes d'Encolpe exposent les monuments de l'épopée au rire : l'attitude de Didon aux Enfers devant Enée devient celle de son sexe désespérément mou! La voix d'Encolpe, lorsqu'il se fait poète, loin de creuser un écart avec le cadre narratif en prose, annule toute distance : tout s'équivaut et, mieux, tout a droit de cité en littérature. C'est ce que revendique le poème suivant d'Encolpe en 132, 15 composé de quatre distiques élégiaques :

\section{Quid me constricta spectatis fronte Catones,} damnatisque nouae simplicitatis opus?

Sermonis puri non tristis gratia ridet, quodque facit populus, candida lingua refert.

«Pourquoi me regarder le sourcil froncé, ô Catons,

Et condamner une œuvre d'une candeur encore jamais vue?

Y sourit la grâce sans austérité d'une langue pure

Et tout ce que fait le peuple y est rapporté en un style limpide. »

Le narrateur explique une partie de ses intentions et des principes littéraires en jeu dans son opus. Les sujets populaires, bas, triviaux, notamment l'amour et ses plaisirs, évités par les genres littéraires nobles, font partie intégrante des matériaux qui nourrissent une œuvre littéraire et ces motifs viennent contaminer toute réminiscence épique. Le narrateur prône une représentation totale du comportement humain, choisit le réalisme de la satire qui ne néglige aucun des aspects les plus bas et les plus cachés de la vie ordinaire, le défend en invoquant Epicure, mais il lui confère une nouvelle et originale fonction. Il ne s'agit plus de dénoncer le vice pour encourager la vertu dans une perspective "moraliste", comme de nombreux critiques ont pu le penser. La peinture réaliste fondée sur une imitation humoristique d'autres genres littéraires a pour seul but le burlesque. Par exemple, le poème en 139, 2 développe le motif de " la lourde colère de Priape ",

17 Bakhtine, The dialogic imagination, 1981, pp. 22-23. 
grauis ira Priapi, qui poursuit Encolpe comme Ulysse l'était par Poséidon dans l'Odyssée. Le rire jaillit, non pas aux dépens d'un genre littéraire ou d'une œuvre particulière, mais aux dépens du seul narrateur Encolpe : l'image de celui-ci en sort particulièrement dégradée. Chaque poème ne fait qu'aggraver la dimension grotesque du personnage. Dans le poème 136,6 dont seulement cinq hexamètres dactyliques ont été conservés, Encolpe réclame le rôle de héros, à l'instar d'Hercule, pour avoir tué une oie qui le chargeait ! Du coup, ses jugements sont subtilement annulés, parce qu'il est luimême la première cible de la satire.

\section{Conclusion}

La voix du narrateur principal Encolpe se révèle, à l'examen, d'une extrême complexité : oscillant entre prose et poésie, elle permet en réalité l'imbrication de différentes " voix », celle d'abord du récit, mais aussi celle du dialogue, celle de la "vignette », celle de la citation, celle du montage de citations, celle enfin, plus impersonnelle, qui commente l'action ou même la finalité de l'œuvre et ses intentions littéraires. Cette multiplicité et cette interaction de divers plans de représentation, cette distribution originale entre plusieurs voix font du Satiricon une des premières manifestations de ce que l'on appellera plus tard un roman "polyphonique ».

Lorsque le narrateur interrompt sa narration pour insérer un poème, il ne fait souvent que réfléchir de manière redondante son récit ou qu'apporter un méta-commentaire qui invite à le lire comme un jeu mimique revendiquant la représentation totale du comportement humain. Dans tous les cas, cette voix plurielle recourt à la technique du « contrepoint » par laquelle tout événement, toute parole, tout personnage semblent appeler leur contraire, leur double parodique. L'entrelacement d'événements vulgaires ou ridicules dans le récit en prose avec des poèmes idéalisants sur l'amour aboutit à un effet discordant que rehausse le commentaire autodépréciatif que fait le narrateur de son propre comportement par trop naïf.

Mais l'élément de rupture que comporte en soi l'insertion de la poésie est aussi un moyen de faire que tout 
s'équivaut, que les distances entre les genres s'abolissent : l'héroïsme national de l'épopée se transforme en obscénités privées. Le narrateur lui-même en perd son identité. Les poèmes d'Encolpe, parce qu'ils retranscrivent des lieux communs rhétoriques, des réminiscences tragiques, épiques, surimposent au récit du narrateur un discours somme toute étranger qui en fait un personnage sans réelle profondeur psychologique.

Signe d'une tension entre deux formes concurrentes, le recours à la prosimétrie ne serait-il pas le moyen pour Pétrone de faire de son œuvre une fiction théâtrale qui explorerait les différentes structures poétiques de représentation pour finalement les parodier et les nier toutes dans une écriture de totale subversion? Une fiction qui, par ses allusions récurrentes au théâtre, voudrait restituer la dimension illusoire de ce monde où le narrateur ne serait qu'un rôle, une persona sur une scène de théâtre. En façonnant un narrateur poète, Pétrone permet à sa fiction de recevoir une identité propre, se construisant avec et contre les autres formes poétiques dans une relation agonistique consciente, en étant ce que ces formes littéraires ne sont pas. 